\title{
FREE VIBRATION TO A SYSTEM OF CANTILEVER NANOBEAMS
}

\author{
Anita Ciekot, Stanisław Kukla \\ Institute of Mathematics, Czestochowa University of Technology \\ Częstochowa, Poland \\ anita.ciekot@im.pcz.pl,stanislaw.kukla@im.pcz.pl
}

\begin{abstract}
This paper presents a solution to the free vibration problem of a system of two cantilever nanobeams. The nanobeams of the system are axially loaded and coupled by discrete translational springs. The solution of the problem by using the Green's function method has been obtained. A numerical example shows the effect of the small scale on free vibration frequencies of the nanobeam system considered.
\end{abstract}

Keywords: cantilever nanobeams, free vibration, Green's function method

\section{Introduction}

The classical theories of vibration of beams at macro-scales are well established, but the vibration behavior of nanostructures is far from being well understood. In the last three decades, many researchers have used the nonlocal elasticity theory to analyze the mechanical behaviors of nanostructures, particularly of nanobeams. The nonlocal theory of elasticity was initiated by Eringen [1].Various theories using nonlocal constitutive relations introduced by Eringen were the subject of the paper by Reddy [2]. In the paper are derived the equations of motion of nanobeams based on the Euler-Bernoulli, Timoshenko, Reddy and Levinson beam theories. The nonlocal beam theories for bending, buckling and vibration of nanobeams were studied by Aydogdu [3] and by Thai [4]. In these papers analytical solutions to the vibration problems of nanobeams are also presented. In the contribution by Eltaher et al. [5] the finite element method for vibration analysis of an Euler-Bernoulli nanobeam was applied. The free vibration of a cantilever nanobeam is reported in the papers by Peddieson et al. [6] and Lim et al. [7].

In this paper the free vibration problem of a system of two coupled cantilever nanobeams is studied. The buckling of a system of two nanobeams coupled by a distributed transverse spring was analyzed by Murmu and Adhikari [8]. In the present paper the subject of the consideration is the vibration of a system of two nanobeams connected by an arbitrary number of discrete translational springs. The solution of the problem was obtained using the Green's function method. 
The small-scale effect on the vibration frequencies of the considered system is numerically investigated.

\section{Formulation of the problem}

The equations of motion for the transverse vibration of two simply-supported nanobeams that are coupled by $n$-discrete transitional springs were presented in paper [9]. Considering the free vibrations to a system of cantilever nanobeams we assume the transverse displacement in the form: $w_{i}(x, t)=\bar{W}_{i}(x) \cdot \cos \omega t, \mathrm{i}=1,2$, where $\omega$ is the circular frequency.

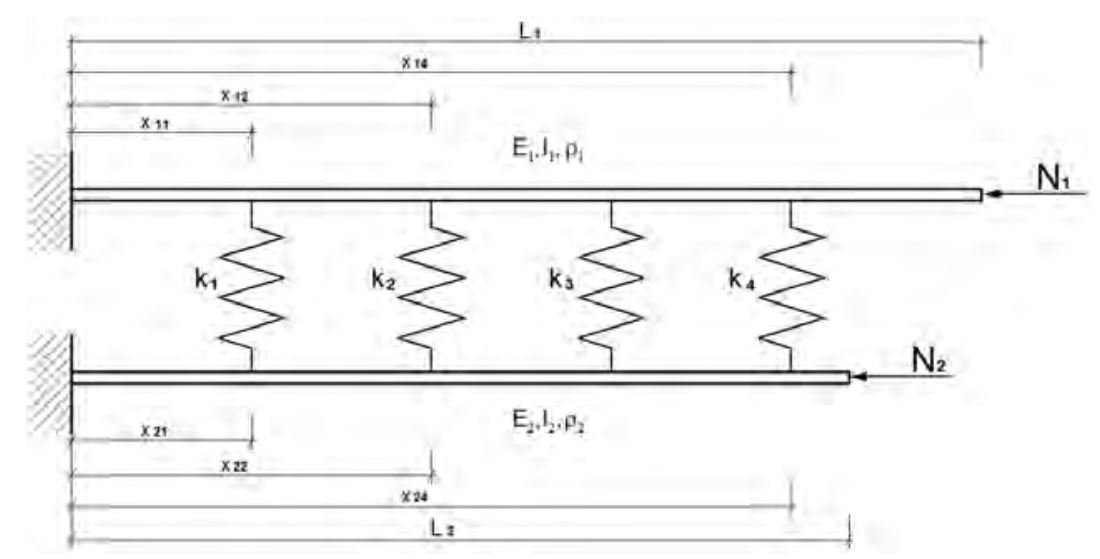

Fig. 1. A sketch of the coupled cantilever nanobeams - system

Moreover, by introducing a new variable $\xi_{i}=\frac{x_{i}}{L}, W_{i}=\frac{\bar{W}_{i}}{L_{i}}$ and $\bar{W}_{i}\left(x_{i}\right)=L_{i} W_{i}\left(\xi_{i}\right)$, the following system of the ordinary differential equations is obtained:

$$
\begin{gathered}
W_{1}^{\prime \prime \prime \prime}\left(\xi_{1}\right)-\bar{F}_{1} W_{1}^{\prime \prime}\left(\xi_{1}\right)-p \Omega^{4} W_{1}\left(\xi_{1}\right)=p \sum_{j=1}^{n} K_{j}\left[W_{1}\left(\xi_{1 j}\right)-\frac{1}{h} W_{2}\left(\xi_{2 j}\right)\right] \delta\left(\xi_{1}-\xi_{1 j}\right) \\
-p \mu^{2} \sum_{j=1}^{n} K_{j}\left[W_{1}^{\prime \prime}\left(\xi_{1 j}\right)-h W_{2}^{\prime \prime}\left(\xi_{2 j}\right)\right] \delta\left(\xi_{1}-\xi_{1 j}\right) \\
W_{2}^{\prime \prime \prime \prime}\left(\xi_{1}\right)-\bar{F}_{2} W_{2}^{\prime \prime}\left(\xi_{2}\right)-q r^{4} \Omega^{4} W_{2}\left(\xi_{2}\right)=-h q s^{2} \sum_{j=1}^{n} K_{j}\left[W_{1}\left(\xi_{1 j}\right)-\frac{1}{h} W_{2}\left(\xi_{2 j}\right)\right] \delta\left(\xi_{2}-\xi_{2 j}\right) \\
+h q s^{2} \mu^{2} \sum_{j=1}^{n} K_{j}\left[W_{1}^{\prime \prime}\left(\xi_{1 j}\right)-h W_{2}^{\prime \prime}\left(\xi_{2 j}\right)\right] \delta\left(\xi_{2}-\xi_{2 j}\right)
\end{gathered}
$$


where: $\quad \Omega^{4}=\frac{\rho_{1} A_{1} L_{1}^{4}}{E_{1} I_{1}} \omega^{2}, \quad K_{j}=\frac{k_{j} L_{1}^{3}}{E_{1} I_{1}}, \quad F_{i}=\frac{N_{i} L_{i}^{2}}{E_{i} I_{i}}, \quad \mu=\frac{e_{0} a}{L_{1}}, \quad s^{2}=\frac{E_{1} I_{1} L_{2}^{3}}{E_{2} I_{2} L_{1}^{3}}$, $r^{4}=\frac{\rho_{2} A_{2} E_{1} I_{1} L_{2}^{4}}{\rho_{1} A_{1} E_{2} I_{2} L_{1}^{4}}, \quad \overline{F_{1}}=\frac{\left(F_{1}+\mu^{2} \Omega^{4}\right)}{\left(1+\mu^{2} F_{1}\right)}, \quad p=\frac{1}{\left(1+\mu^{2} F_{1}\right)}, \quad q=\frac{1}{1+\mu^{2} h^{2} F_{2}}, \quad h=\frac{L_{1}}{L_{2}} ;$ $\bar{F}_{2}=\frac{F_{2}+\mu^{2} r^{4} \Omega^{4} \frac{L_{1}^{2}}{L_{2}^{2}}}{1+\mu^{2} \frac{L_{1}^{2}}{L_{2}^{2}} F_{2}}$.

This system of equations has been solved for boundary conditions which are satisfied by the functions $W_{1}$ and $W_{2}$ :

$$
\begin{gathered}
W_{i}(0)=W_{i}^{\prime}(0)=0 ; \quad i=1,2 \\
W_{i}^{\prime \prime}\left(L_{i}\right)=W_{i}^{\prime \prime \prime}\left(L_{i}\right)-\bar{F}_{i} W_{i}^{\prime}\left(L_{i}\right)=0 \quad i=1,2
\end{gathered}
$$

In order to solve the boundary problem (1)-(4) the Green's function method can be used. The Green's functions $G_{i}$ we find as a solution of the differential equation

$$
\frac{\partial^{4} G_{i}}{\partial \xi_{i}^{4}}-\bar{F}_{i} \frac{\partial^{2} G_{i}}{\partial \xi_{i}^{2}}-\lambda_{i}^{4} G_{i}\left(\xi_{i}\right)=\delta\left(\xi_{i}-\eta_{i}\right)
$$

where $\lambda_{1}=\Omega \sqrt[4]{p}, \lambda_{2}=r \Omega \sqrt[4]{q}$. The functions satisfy the homogenous boundary conditions analogues to the conditions (3)-(4):

$$
\begin{gathered}
G_{i}\left(0, \eta_{i}\right)=\left.\frac{\partial G_{i}}{\partial \xi_{i}}\right|_{\xi_{i}=0}=0 \\
\left.\frac{\partial^{2} G_{i}}{\partial \xi_{i}^{2}}\right|_{\xi_{i}=L_{i}}=\left.\left(\frac{\partial^{3} G_{i}}{\partial \xi_{i}^{3}}-\bar{F}_{i} \frac{\partial G_{i}}{\partial \xi_{i}}\right)\right|_{\xi_{i}=L_{i}}=0
\end{gathered}
$$

Derivation of the Green's functions is presented in Section 3.

\section{The solution to the problem}

Using the Green's function method the solution of the problem (1)-(4) can be presented in the form: 


$$
\begin{gathered}
W_{1}\left(\xi_{1}\right)=p \sum_{j=1}^{n} K_{j}\left[W_{1}\left(\xi_{1 j}\right)-\frac{1}{h} W_{2}\left(\xi_{2 j}\right)\right] G_{1}\left(\xi_{1}, \xi_{1 j}\right) \\
-p \mu^{2} \sum_{j=1}^{n} K_{j}\left[W_{1}^{\prime \prime}\left(\xi_{1 j}\right)-h W_{2}^{\prime \prime}\left(\xi_{2 j}\right)\right] G_{1}\left(\xi_{1}, \xi_{1 j}\right) \\
W_{2}\left(\xi_{2}\right)=-h q s^{2} \sum_{j=1}^{n} K_{j}\left[W_{1}\left(\xi_{1 j}\right)-\frac{1}{h} W_{2}\left(\xi_{2 j}\right)\right] G_{2}\left(\xi_{2}, \xi_{2 j}\right) \\
+h q s^{2} \mu^{2} \sum_{j=1}^{n} K_{j}\left[W_{1}^{\prime \prime}\left(\xi_{1 j}\right)-h W_{2}^{\prime \prime}\left(\xi_{2 j}\right)\right] G_{2}\left(\xi_{2}, \xi_{2 j}\right)
\end{gathered}
$$

Substituting $\xi_{1}=\xi_{1 j}, \xi_{2}=\xi_{2 j},(j=1,2, \ldots n)$ in equations (8)-(9) and in equations which express the second order derivatives of the functions $W_{1}\left(\xi_{1}\right)$ and $W_{2}\left(\xi_{2}\right)$, a system of equations is obtained. This equation system can be written in the following matrix form:

$$
\mathbf{Y} \cdot \mathbf{x}=\mathbf{0}
$$

where

$$
\mathbf{Y}=\left[\begin{array}{cccc}
p \mathbf{A}-\mathbf{E} & -\frac{p}{h} \mathbf{A} & -p \mu^{2} \mathbf{A} & h p \mu^{2} \mathbf{A} \\
-h q s^{2} \mathbf{B} & q s^{2} \mathbf{B}-\mathbf{E} & h q s^{2} \mu^{2} \mathbf{B} & -h^{2} q s^{2} \mu^{2} \mathbf{B} \\
p \mathbf{C} & -\frac{p}{h} \mathbf{C} & -p \mu^{2} \mathbf{C}-\mathbf{E} & h p \mu^{2} \mathbf{C} \\
-h q s^{2} \mathbf{D} & q s^{2} \mathbf{D} & h q s^{2} \mu^{2} \mathbf{D} & -h^{2} q s^{2} \mu^{2} \mathbf{D}-\mathbf{E}
\end{array}\right]
$$

$\mathbf{A}=\left[a_{i j}\right], \quad \mathbf{B}=\left[b_{i j}\right], \quad \mathbf{C}=\left[c_{i j}\right], \quad \mathbf{D}=\left[d_{i j}\right], \quad a_{i j}=K_{j} G_{1}\left(\xi_{1 i}, \xi_{1 j}\right)$, $b_{i j}=K_{j} G_{2}\left(\xi_{2 i}, \xi_{2 j}\right), \quad c_{i j}=K_{j} G_{1} "\left(\xi_{1 i}, \xi_{1 j}\right), d_{i j}=K_{j} G_{2} "\left(\xi_{2 i}, \xi_{2 j}\right), \quad i, j=1,2, \ldots, n$, $\mathbf{X}=\left[W_{1}\left(\xi_{11}\right) \cdots W_{1}\left(\xi_{1 n}\right) W_{2}\left(\xi_{21}\right) \cdots W_{2}\left(\xi_{2 n}\right) W_{1}^{\prime \prime}\left(\xi_{11}\right) \cdots W_{1}^{\prime \prime}\left(\xi_{1 n}\right) W_{2}^{\prime \prime}\left(\xi_{21}\right) \cdots W_{2}^{\prime \prime}\left(\xi_{2 n}\right)\right]^{T}$ and $\mathbf{E}$ denotes an identity matrix.

The non-trivial solutions of equation (10) exist for these values of the $\Omega$ for which the determinant of the matrix $\mathbf{Y}$ is equal to zero.

$$
\operatorname{det} \mathbf{Y}=\mathbf{0}
$$

Using the properties of the determinant this equation can be also written in the form: 


$$
\operatorname{det}\left[\begin{array}{cccc}
-\mathbf{E} & \mathbf{0} & \mathbf{0} & h p \mu^{2} \mathbf{A} \\
\mathbf{0} & -\mathbf{E} & \mathbf{0} & -h^{2} \mu^{2}\left(p \mathbf{A}+q s^{2} \mathbf{B}\right) \\
\mathbf{0} & \mathbf{0} & -\mathbf{E} & -h\left(p \mathbf{A}+q s^{2} \mathbf{B}-p \mu^{2} \mathbf{C}\right) \\
\mathbf{0} & \mathbf{0} & \mathbf{0} & p \mathbf{A}+q s^{2} \mathbf{B}-p \mu^{2} \mathbf{C}-h^{2} q s^{2} \mu^{2} \mathbf{D}-\mathbf{E}
\end{array}\right]=0
$$

or

$$
\operatorname{det}\left(p \mathbf{A}+q s^{2} \mathbf{B}-p \mu^{2} \mathbf{C}-h^{2} q s^{2} \mu^{2} \mathbf{D}-\mathbf{E}\right)=0
$$

Finally we have

$$
\operatorname{det}(\mathbf{M}-\mathbf{E})=0
$$

where $\mathbf{M}=\left[M_{i j}\right]_{1 \leq i, j \leq n}$, and

$M_{i j}=K_{j}\left[p G_{1}\left(\xi_{1 i}, \xi_{1 j}\right)+q s^{2} G_{2}\left(\xi_{2 i}, \xi_{2 j}\right)-\mu^{2}\left(p G_{1} "\left(\xi_{1 i}, \xi_{1 j}\right)+q h^{2} s^{2} G_{2} "\left(\xi_{2 i}, \xi_{2 j}\right)\right)\right]$

The roots $\Omega_{k}(k=1,2, \ldots)$ of equation (13) are the natural frequencies of the nanobeam system and can be determined numerically.

\section{Derivation of the Green's functions}

The Green's functions $G_{i}(\xi, \eta)$ are solutions of the differential equation (5). Derivation of the Green's functions which are necessary in the considered problem is analogous to those presented in the paper [8] for simply supported nanobeams. The general solution of the equation (5) can be written in the form (index $i$ is omitted):

$$
G(\xi-\eta)=C_{1} \cos \alpha \xi+C_{2} \sin \alpha \xi+C_{3} \operatorname{ch} \beta \xi+C_{4} \operatorname{sh} \beta \xi+G_{0}(\xi-\eta) \cdot H(\xi-\eta)
$$

where $\alpha=\sqrt{\frac{1}{2}\left(\sqrt{F_{i}^{2}+4 \Omega^{4}}-F_{i}\right)}, \quad \beta=\sqrt{\frac{1}{2}\left(\sqrt{F_{i}^{2}+4 \Omega^{4}}+F_{i}\right)}, H(\cdot)$ denotes the Heaviside function and

$$
G_{0}(u)=-\frac{1}{\alpha^{2}+\beta^{2}}\left(\frac{1}{\alpha} \sin \alpha u-\frac{1}{\beta} \sinh \beta u\right)
$$

The constants $C_{1}, C_{2}, C_{3}$ and $C_{4}$ occurring in equation (14) are determined using boundary conditions (6)-(7). After some transformations we obtain 


$$
\begin{gathered}
C_{1}=\frac{1}{w}\left(G_{0}^{\prime \prime}(L-\xi) \cdot \Phi^{\prime}(L)-G_{0}^{\prime \prime \prime}(L-\xi) \cdot \Phi(L)\right) \\
C_{2}=\frac{1}{w}\left(G_{0}^{\prime \prime \prime}(L-\xi) \cdot \Phi^{\prime}(L)-\Phi^{\prime \prime \prime}(L) \cdot G_{0}^{\prime \prime}(L-\xi)\right) \\
C_{3}=-C_{1}, \quad C_{4}=\frac{-\alpha}{\beta} \cdot C_{2}
\end{gathered}
$$

where

$$
\begin{aligned}
& w=\Phi^{\prime 2}(L)-\Phi(L) \cdot \Phi^{\prime \prime} "(L) \\
& \Phi(x)=\alpha \cdot \sin \alpha x+\beta \cdot \operatorname{sh} \beta x
\end{aligned}
$$

Finally, the Green's function is given by equation (14) where the function $G_{0}$, the constants $C_{1}, C_{2}, C_{3}, C_{4}, w$ and function $\Phi$ are given by equations (15)-(20). The formulas are used in numerical calculations of the Green's function.
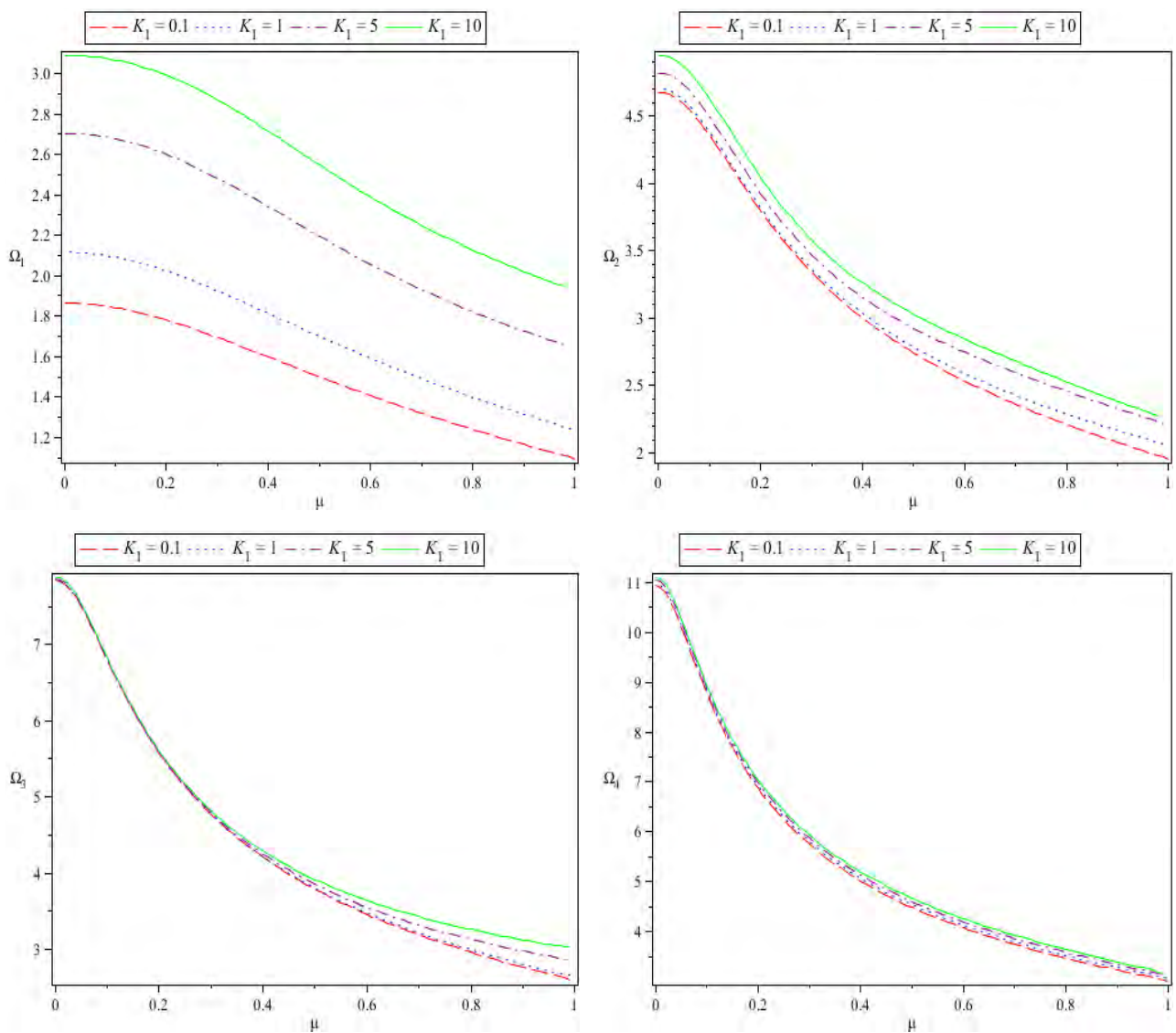

Fig. 2. The first four dimensionless natural vibration frequencies as a function of $\mu$ 


\section{Numerical example}

The frequency equation of the system of nanobeams derived in an analytical form has been used to numerical investigation of the effect of small scale on the eigenfrequencies of this system. In computations the system of two nanobeams of identical length and the same physical properties has been assumed which were axially loaded $\left(F_{1}=F_{2}=0.25\right)$. The nanobeams are coupled by two translational springs characterized by the same spring stiffness coefficient. One of the springs connects the middle of the nanobeams and second connects the free nanobeam ends. The calculations were performed for four different values of spring stiffness coefficient: $K_{1}=0.1 ; 1.0 ; 5.0 ; 10.0$. The four dimensionless vibration frequencies as functions of parameter $\mu$ are plotted in Figure 2. The computations have been performed using the Maple package [10]. The figures show that as the parameter $\mu$ increases, the free vibration frequencies decrease for all spring stiffness coefficients considered. The spring stiffness significantly influences the first eigenfrequency $\Omega_{1}$ and their effect is small for the higher frequencies.

\section{Summary}

The solution of the free translational vibration problem of the system of two cantilever nanobeams connected by discrete springs has been presented. The Green's function method was applied to obtain the eigenfrequency equation and next the method of bisection was used to numerically determine the free vibration frequencies. The effect of the small scale and the spring stiffness coefficient on the eigenfrequencies of the nanobeam system has been shown graphically. Although the number of coupling springs taken into account in the presented example was limited to two, the derived eigenequation includes the case of the system of nanobeams connected by an arbitrary number of discrete springs.

\section{References}

[1] Eringen A.C., On differential equations of nonlocal elasticity and solutions of screw dislocation and surface-waves, Journal of Applied Physics 1983, 54, 4703-4710.

[2] Reddy J.N., Nonlocal theories for bending, buckling and vibration of beams, International Journal of Engineering Science 2007, 45, 228-307.

[3] Aydogdu M., A general nonlocal beam theory: its application to nanobeam bending, buckling and vibration, Physica E 2009, 41, 1651-1655.

[4] Thai H.T., A nonlocal beam theory for bending, buckling and vibration of nanobeams, International Journal of Engineering Science 2012, 52, 56-64.

[5] Eltaher M.A., Alshorbagy A.E., Mahmoud F.F., Vibration analysis of Euler-Bernoulli nanobeams by using finite element method, Applied Mathematical Modelling 2013, 3(7), 4787-4797.

[6] Peddieson J., Buchanan G.R., McNitt R.P., Application of nonlocal continuum models to nanotechnology, International Journal of Engineering Science 2003, 41, 305-312. 
[7] Lim C.W., Li C., Lu J.L., The effects of stiffness strengthening nonlocal stress and axial tension on free vibration of cantilever nanobeams, Interaction and Multiscale Mech. 2009, 2(3), 223-233.

[8] Murmu T., Adhikari S., Axial instability of double-nanobeam-system, Physics Letters A 2011, 375, 601-608.

[9] Ciekot A., Kukla S., Frequency analysis of a double-nanobeam system, Journal of Applied Mathematics and Computational Mechanics 2014, 13(1), 23-31.

[10] Richards D., Advanced Mathematical Methods with Maple, Cambridge University Press, 2009. 